\title{
Characteristics of the Third COVID-19 Pandemic Wave with Special Focus on Socioeconomic Inequalities in Morbidity, Mortality and the Uptake of COVID-19 Vaccination in Hungary
}

\author{
Beatrix Oroszi ${ }^{1}$, Attila Juhász ${ }^{2} \mathbb{D}$, Csilla Nagy ${ }^{2}$, Judit Krisztina Horváth ${ }^{1}$, Krisztina Eszter Komlós ${ }^{1}$ (D), \\ Gergő Túri ${ }^{1}$ (D), Martin McKee ${ }^{3, *}$ and Róza Ádány ${ }^{4,5, *(D)}$
}

check for updates

Citation: Oroszi, B.; Juhász, A.; Nagy, C.; Horváth, J.K.; Komlós, K.E.; Túri,

G.; McKee, M.; Ádány, R.

Characteristics of the Third COVID-19 Pandemic Wave with Special Focus on Socioeconomic Inequalities in Morbidity, Mortality and the Uptake of COVID-19 Vaccination in Hungary. J. Pers. Med. 2022, 12, 388. https://doi.org/ 10.3390/jpm12030388

Academic Editor: Reginald M. Gorczynski

Received: 4 February 2022

Accepted: 1 March 2022

Published: 3 March 2022

Publisher's Note: MDPI stays neutral with regard to jurisdictional claims in published maps and institutional affiliations.

Copyright: (c) 2022 by the authors. Licensee MDPI, Basel, Switzerland. This article is an open access article distributed under the terms and conditions of the Creative Commons Attribution (CC BY) license (https:/ / creativecommons.org/licenses/by/ $4.0 /)$.
1 Epidemiology and Surveillance Centre, Semmelweis University, 25. Üllői Street, 1085 Budapest, Hungary; oroszi.beatrix@semmelweis-univ.hu (B.O.); horvath.judit_krisztina@semmelweis-univ.hu (J.K.H.); komlos.krisztina_eszter@semmelweis-univ.hu (K.E.K.); turi.gergo@semmelweis-univ.hu (G.T.)

2 Department of Public Health, Government Office of Capital City Budapest, 174. Váci Avenue, 1138 Budapest, Hungary; juhasz.attila@kmr.antsz.hu (A.J.); nagy.csilla@kmr.antsz.hu (C.N.)

3 European Centre on Health of Societies in Transition (ECOHOST), London School of Hygiene and Tropical Medicine, Keppel Street, London WC1E 7HT, UK

4 MTA-DE-Public Health Research Group, Department of Public Health and Epidemiology, Faculty of Medicine, University of Debrecen, 26. Kassai Street, 4028 Debrecen, Hungary

5 Department of Public Health, Faculty of Medicine, Semmelweis University, 25. Üllői Street, 1085 Budapest, Hungary

* Correspondence: martin.mckee@lshtm.ac.uk (M.M.); adany.roza@med.unideb.hu (R.Á.); Tel.: +44-7973-832576 (M.M.); +36-30-9676558 (R.Á.)

\begin{abstract}
Governments are increasingly looking to vaccination to provide a path out of the COVID-19 pandemic. Hungary offers an example to investigate whether social inequalities compromise what a successful vaccine program can achieve. COVID-19 morbidity, mortality, and vaccination coverage were characterized by calculation of indirectly standardized ratios in the Hungarian population during the third pandemic wave at the level of municipalities, classified into deprivation quintiles. Then, their association with socioeconomic deprivation was assessed using ecological regression. Compared to the national average, people living in the most deprived municipalities had a $15-24 \%$ lower relative incidence of confirmed COVID-19 cases, but a 17-37\% higher relative mortality and a $38 \%$ lower vaccination coverage. At an ecological level, COVID-19 mortality showed a strong positive association with deprivation and an inverse association with vaccination coverage $\left(\mathrm{RR}_{\text {Vaccination }}=0.86(0.75-0.98)\right)$, but the latter became non-significant after adjustment for deprivation $\left(R R_{\text {Vaccination }}=0.95(0.84-1.09), R_{\text {Deprivation }}=1.10(1.07-1.14)\right)$. Even what is widely viewed as one of the more successful vaccine roll outs was unable to close the gap in COVID-19 mortality during the third pandemic wave in Hungary. This is likely to be due to the challenges of reaching those living in the most deprived municipalities who experienced the highest mortality rates during the third wave.
\end{abstract}

Keywords: COVID-19; morbidity; mortality; excess mortality; vaccination coverage; deprivation; socioeconomic inequities; Roma

\section{Introduction}

A government developing a response to a pandemic can employ a variety of approaches [1,2]. Some, such as restrictions on facilities where people mix or face mask mandates, seek to reduce transmission. Others, such as vaccine programmes, seek to increase immunity [3-6]. Additionally, others, such as financial support for those unable to work, offer help to those affected by the disease and necessary responses. The evidence, from empirical studies and models, points to a combination of measures as most likely to control disease spread and minimize collateral harms [7-10]. 
More restrictive measures to decrease population mixing and contact numbers often cause the biggest problems for those already disadvantaged, who cannot work from home, may work in the informal economy, live in overcrowded dwellings, and have few resources on which they can draw [11]. Those taking this perspective argue that relying on vaccines is less likely to exacerbate inequalities. However, inequities in the uptake of COVID-19 vaccination, which are reported from several countries, may jeopardize this goal [12,13].

During the 2020-2021 winter season, the new, fast-spreading Alpha variant appeared and gradually displaced the previous dominant variant in Europe [14,15]. In Hungary, the second wave and accompanying restrictions were not over when the even bigger third Alpha wave arrived at the beginning of 2021. This created an imperative to make rapid progress with the mass vaccination program, supplementing the four vaccines approved by the European Medicines Agency with Gamaleya Gam-COVID-Vac/Sputnik V and Beijing/Sinopharm BBIBP-CorV, granted emergency approval in Hungary. In Hungary, until the end of the third wave, 4,846,431 people became fully vaccinated (with a single dose of the Janssen vaccine or two doses of the others). A total of $61.3 \%$ received one of the four vaccines approved by the European Medicines Agency (41.7\% Pfizer/BioNTech, 10.9\% AstraZeneca, 6.6\% Moderna and 2.1\% Janssen), and 38.7\% were vaccinated with Sputnik V (18.2\%) or Sinopharm (20.5\%) [16]. At the beginning of July 2021, Hungary had fully vaccinated a higher share of its population than any EU country except Malta, and higher than the UK, widely seen as an early leader [17]. Hungary is fortunate in having granular data on not only population characteristics but also vaccine uptake and COVID-19 epidemiology. We have previously taken advantage of this to analyze the association between these characteristics and patterns of COVID-19 during the second wave, finding that those in more deprived areas were less likely to be diagnosed with COVID-19 but more likely to die [18]. Here, we build on this earlier analysis by analyzing the epidemiology of the third wave, which occurred soon after the vaccination program had been rolled out.

Our study has several linked components. First, we describe the morbidity and mortality of the third pandemic wave, and we assess the spatial association between socioeconomic characteristics of the population and morbidity and mortality due to COVID-19. Then, we compare the second and third pandemic waves concerning the spatial distribution of confirmed COVID-19 cases and deaths. We then analyze the spatial distribution of vaccination coverage and its association with deprivation. Finally, we explore the interaction between vaccination coverage, mortality, and deprivation at the end of the third wave. In this way, we can look at the health consequences of relying primarily on mass vaccination, which had the benefit of speed but did not take account of the challenge of addressing inequalities. Unlike other studies that have used entire countries as the unit of analysis, this study exploits the substantial diversity within Hungary.

\section{Methods}

\subsection{Data Collection}

The Hungarian Notifiable Disease Surveillance System, operated by the National Public Health Center (NPHC), provided data on registered COVID-19 cases. Cases with laboratory confirmation (detection of SARS-CoV-2 by polymerase chain reaction (PCR) or SARS-CoV-2 antigen by a lateral flow test), irrespective of clinical signs and symptoms, were considered a confirmed case [19] and were involved in this study. The epidemic curve used the date of laboratory confirmation. Data on age, sex, municipality of residence, and outcome (alive/dead) were also available on an individual level.

Hungary is divided into 23 districts in Budapest and 197 in the 19 counties (the Hungarian names of these types of districts differ but can be considered the same for this analysis). Districts in counties, but not the capital, are further divided into a total of 3155 municipalities. Data on the share of Roma in the population were available only at the district level, while all other data were available at the municipality level (except in Budapest, where the lowest administrative level is the district). 
Vaccination data (on doses administered in Hungary between week 52, 2020 and week 29, 2021) were provided by the National Health Insurance Fund of Hungary at the level of municipalities, by 5-year age groups, not broken down by sex.

The first and last days of the third pandemic wave in Hungary were defined as the days before and after the peak of the third wave, when the lowest daily case rate was recorded, in accordance with a previous study [18]. These days were 25 January 2021 and 4 July 2021.

Morbidity and mortality were defined as the incidence of COVID-19 cases or deaths, respectively, confirmed during the third wave. Cause of deaths was defined based on previous confirmation of COVID-19 by PCR or antigen test and on listing it as the underlying or a contributing cause of death.

Data on all-cause mortality for the third wave were obtained through the national Electronic Civil Registration System, which allowed us to investigate time trends by sex, age, and week of death. Excess mortality was calculated with reference to the years compared with the 2014-2019 mortality on data available as of 18 October 2021. The Central Office for Administrative and Electronic Public Services provided population data by sex and age at the municipality level for the same period.

As noted above, we have previously published data on the second pandemic wave (22 June 2020 and 24 January 2021) [18].

\subsection{Deprivation Index}

The area-based composite deprivation index [20], used successfully in several previous studies [18,21-26], provided information on socioeconomic deprivation at the municipality level. The index combined seven socioeconomic indicators (income, education level, unemployment rate, single-parent and large family households, housing density and car ownership) obtained from the databases of the Hungarian Central Statistical Office (2011 Census) and the Hungarian Tax and Financial Control Office (2011). Weights were estimated using principal component analysis. Higher values of the index correspond to lower socioeconomic status.

\subsection{Statistical Analysis}

Excess weekly mortality relative to the corresponding weeks in 2014-2019 were calculated for the third pandemic wave, from week 8 to week 18 of 2021, using the Rapid Inquiry Facility (RIF) [27] and applying age (5-year age groups) and sex-specific rates in the Hungarian population.

Consistent with our earlier study, we conducted a hierarchical Bayesian analysis $[18,27,28]$. The smoothed indirectly standardized ratios were estimated using the Integrated Nested Laplace Approximation (INLA) method [29] with expected cases adjusted for age (by 5-year age groups), sex and vaccination rates by age (again 5-year age groups). On the maps, areas were considered to have significantly high or low relative ratios if the estimated risk values differ from 1 with at least $80 \%$ probability [30].

Spatial scan statistics were applied to identify age, and sex-adjusted morbidity, mortality, and vaccination clusters with elevated or reduced ratios, confirming and complementing the results of disease mapping $[27,31]$. Fifty percent of the population at risk was defined as the maximum spatial cluster size.

We used the risk analysis module of RIF to assess the association between deprivation and morbidity, mortality (sex-specific) and vaccination coverage [27]. Municipalities were categorized into deprivation quintiles and indirect standardized ratios were calculated for each band. Chi-square tests for homogeneity and linearity trends were used to assess the overall association with deprivation.

Hungary, like other central European countries, has a large Roma population that experiences high levels of deprivation and worse health status and access to care. Thus, we looked in detail at municipalities in the 10 districts with the highest proportion of 
Roma in the population (27.9-39.0\%), as identified in a previous nationwide survey by Pénzes et al. [32,33].

\subsection{Shared Component Model}

The spatial similarity of morbidity and mortality due to COVID-19 between the second and third pandemic waves was assessed using the shared component model. A "centered" version of the full Bayesian spatial shared component disease model proposed by Knorr-Held and Best [34] was applied to explore spatial risk patterns using conditional autoregression priors for the latent components to capture the spatial distribution of shared component [35]. The aim of our analysis was to identify the spatial pattern of the shared component, so we included only spatially structured random effects for the shared and time-specific latent components.

Expected case numbers were defined based on age- (by 5-year age groups) and sex-specific rates of the Hungarian population for the second and the third pandemic wave, respectively.

The models were fitted to the data using full Bayesian estimation and implemented in WinBUGS software [36]. Two different chains were run, and convergence was assessed using Gelman-Rubin's convergence diagnostics [37]. The first 500,000 samples were discarded as burn-in and we ran a further 500,000 iterations, keeping every 50th, which were used in the calculation of the posterior estimates. The posterior probability of each area having an average risk above one ( $R R>1)$ was computed as a measure of uncertainty and areas with high or low relative ratios were marked, where the estimated risk values were at least $80 \%$ likely to differ from 1 [30].

\subsection{Ecological Regression}

An ecological regression model was used to investigate simultaneously spatial relationships between COVID-19 vaccination, deprivation, and COVID-19 mortality. By extending the disease mapping models [28] with the spatial risk factor, we can take into account the assumed effect of residual spatial autocorrelation as an 'unmeasured risk factor'. Ignoring this component may lead to biased estimates of regression coefficients, or underestimation of uncertainty by falsely estimating narrower confidence intervals [38].

The association between COVID-19-related mortality and vaccination coverage, controlling for deprivation as a confounder, was investigated at the municipality level by ecological regression using the INLA method [29]. The collinearity of the regression model was assessed by determining the variance inflation factor (VIF) [39]. The Deviance Information Criterion (DIC) and the Watanabe-Akaike Information Criterion (WAIC) were applied to select the best-fitting model from a variety of models $[40,41]$.

\section{Results}

During the third pandemic wave, the cumulative number of reported confirmed COVID-19 cases was 447,966 (4529 cases per 100,000 population), while the reported number of deaths was 15,494 (crude case-fatality ratio 3.5\%) (recorded and validated until 25 October 2021) (Figure 1). Although the duration of the third wave was shorter than that of the second wave, the cumulative number of cases reported was $25.8 \%$ higher than during the second wave (356,194 cases, 3601 cases per 100,000 population).

The daily number of newly registered cases started to increase during the last week of January 2021, followed by an increase in COVID-19-related deaths 3 weeks later (Figure 1). The peak of confirmed new cases was recorded on 25 March (11,266 or 114 per 100,000), and that of deaths (by date of death) on 1 April $(289$, or 3 per 100,000). At the peak of the third wave, the number of new cases $(11,266)$ was $65.3 \%$ higher than at the peak of the second wave (6817). 


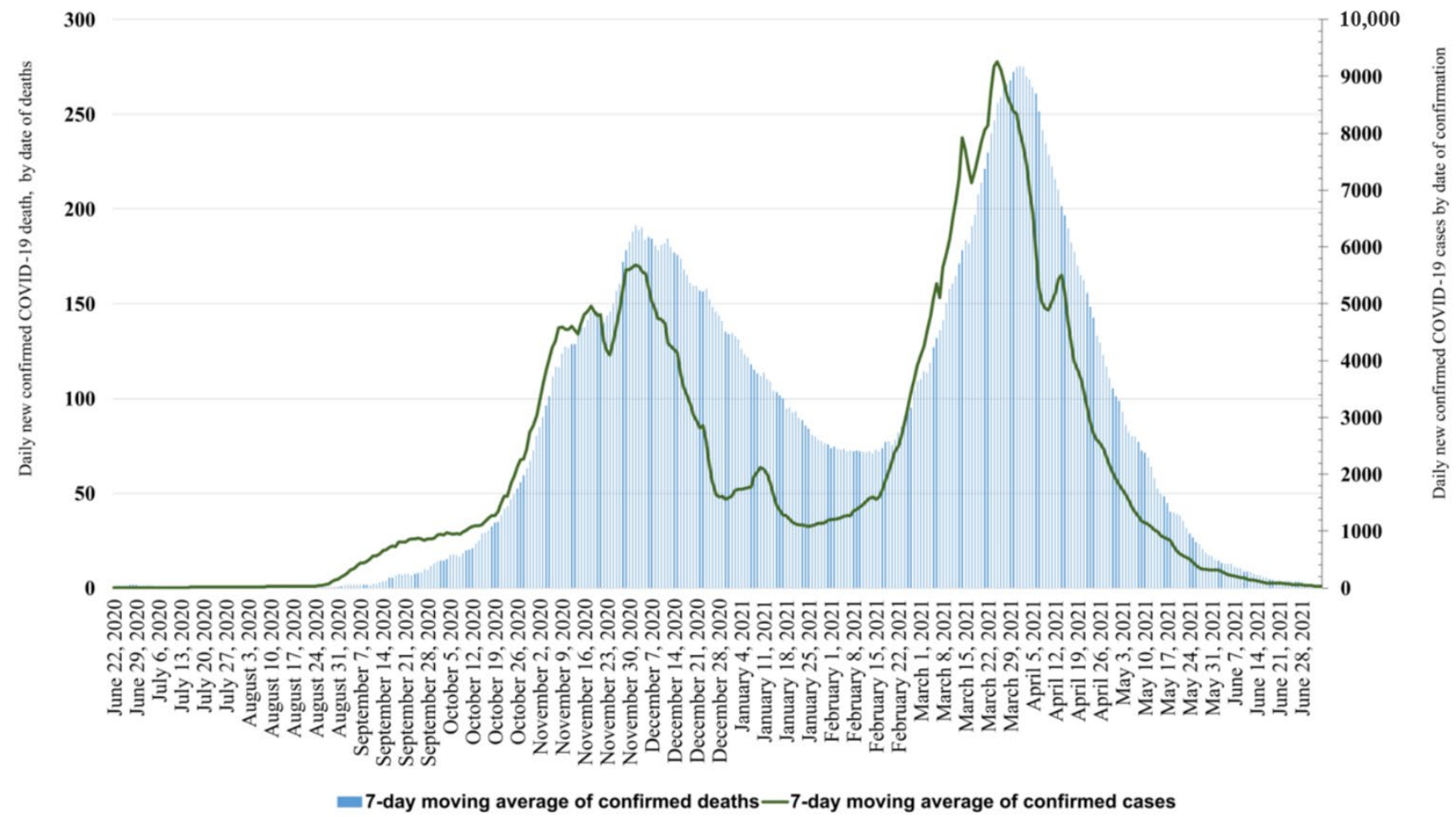

Figure 1. Daily number of confirmed COVID-19 cases (by date of confirmation) and deaths (by date of death) during the second (22 June 2020-24 January 2021) and the third (25 January 2021-4 July 2021) pandemic waves in Hungary.

The highest crude case-fatality ratio was observed in the $80+$ years age group (30.9\%), followed by the $65-79$ years $(12.5 \%)$ and the $50-64$ years age group $(2.7 \%)$. A total of five deaths were reported among those under 18 years (two under 1 year, and three aged between 14 and 16 years). Among deaths, $76.4 \%$ (11,830 deaths) were in the $65+$ years age group.

Of the 447,966 cases, $52.9 \%(236,797)$ were female and $47.1 \%(211,169)$ male. Confirmed cases among women were higher ( 4626 vs. 4426 per 100,000 population); men were 0.96 ( $95 \%$ CI 0.95 to 0.96 ) as likely to be ascertained as a COVID-19 case. However, men had 1.2 ( $95 \%$ CI 1.15 to 1.23 ) times the risk of death (171 cases vs. 143 cases per 100,000).

During the third COVID-19 pandemic wave, excess mortality from all causes started to increase steeply after week 8,2021 . Between week 8 and 19 , significant excess deaths were found in all age groups (excess mortality ${ }_{0-\mathrm{X}}$ years: 1.32 (CI: 1.33-1.34), excess number of death $0-\mathrm{X}$ years: 10,633 (CI: 10,326-10,937); excess mortality50-64 years: 1.40 (CI: 1.39-1.42), excess number of death ${ }_{50-64 \text { years }} 2093$ (CI: 1970-2212); excess mortality ${ }_{65-X}$ years: 1.32 (CI: 1.30-1.33), excess number of death ${ }_{65-X}$ years: 7994 (CI: 7720-8265)) (Figure 2A-C). In total, excess mortality was $32 \%$ higher than the average weekly mortality for the period 2014-2019 for all ages, 40\% higher for 50-64 years, and 32\% higher for 65 years or over (Figure 2A-C).

The relative excess mortality was the highest on week 13 in all age groups (for age $0-X$ years $1.69(1.64-1.74))$ and for 50-64 years, $1.77(1.66-1.90)$, as well as in the 65 years or over age groups (1.65 (1.59-1.71)). Compared to the 2014-2019 averages in corresponding weeks, excess mortality was $69 \%, 77 \%$ and $65 \%$ higher, respectively (Figure $2 \mathrm{~A}-\mathrm{C}$ ). 

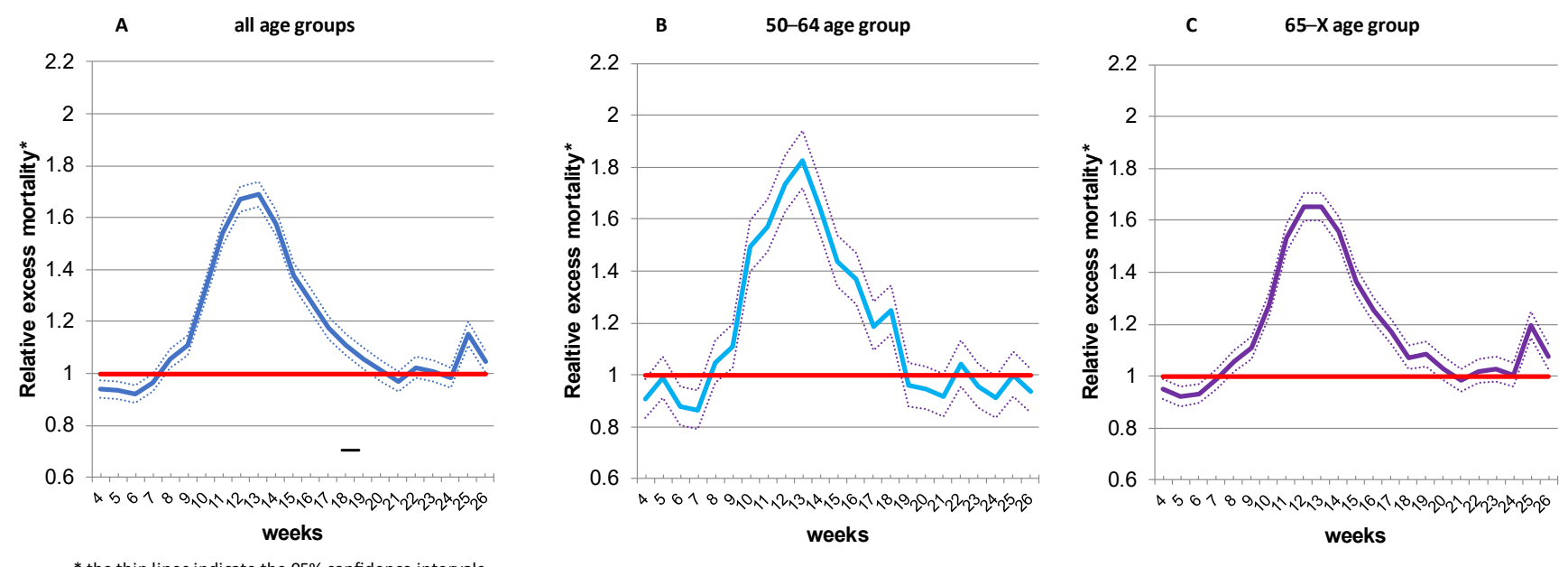

Figure 2. Relative excess mortality due to all causes of death during the third COVID-19 pandemic wave, compared to the average weekly mortality for the period 2014-2019 for all age groups (A), for 50-64 age groups (B) and for 65-X age groups (C) in Hungary.

The vaccination campaign started on week 52, 2020 with the Pfizer-BioNTech/BNT162b2 COVID-19 vaccine. The Moderna/mRNA-1273 COVID-19 vaccine became available from week 2, while the Oxford/AstraZeneca ChAdOx1-S and Gamaleya Gam-COVIDVac/Sputnik V from week 6, the Beijing/Sinopharm BBIBP-CorV from week 8, and the Janssen/Ad26.COV2.S COVID-19 vaccine (also referred to as the Johnson \& Johnson vaccine) from week 18, 2021.

The vaccination campaign ran in parallel with the third pandemic wave in Hungary. At the peak of the epidemic (week 12, 2021), the uptake of the first dose was only $15.0 \%$ in those aged 18 and above and the proportion of fully vaccinated (who had received the second dose) reached $7.2 \%$. By the end of the second wave (week 26, 2021), 59.3\% of this age group was fully vaccinated, with $5.1 \%$ only partially vaccinated (Figure 3 ).

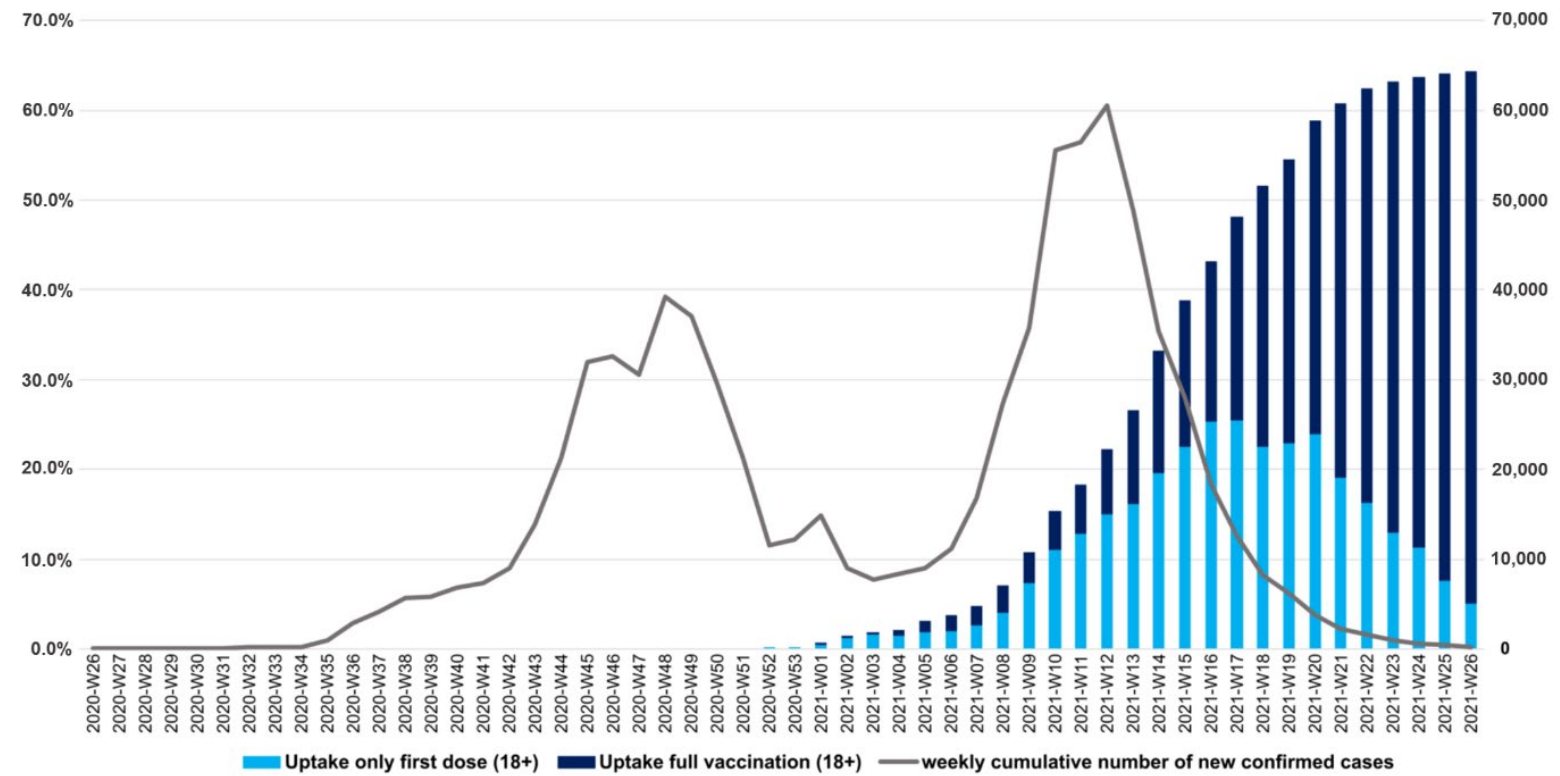

Figure 3. Cumulative uptake (\%) of the first dose and full vaccination in Hungary and weekly number of confirmed cases between week 26, 2020 and week 26, 2021.

When we look at the spatial distribution of COVID-19 vaccination uptake by the end of the third wave, we can see significantly higher vaccination coverage in the western part 
of the capital, Budapest, in county towns and in larger cities. Significantly lower relative vaccination coverage was observed in the more sparsely populated areas of north-eastern and south-western Hungary (Figure 4A,B).

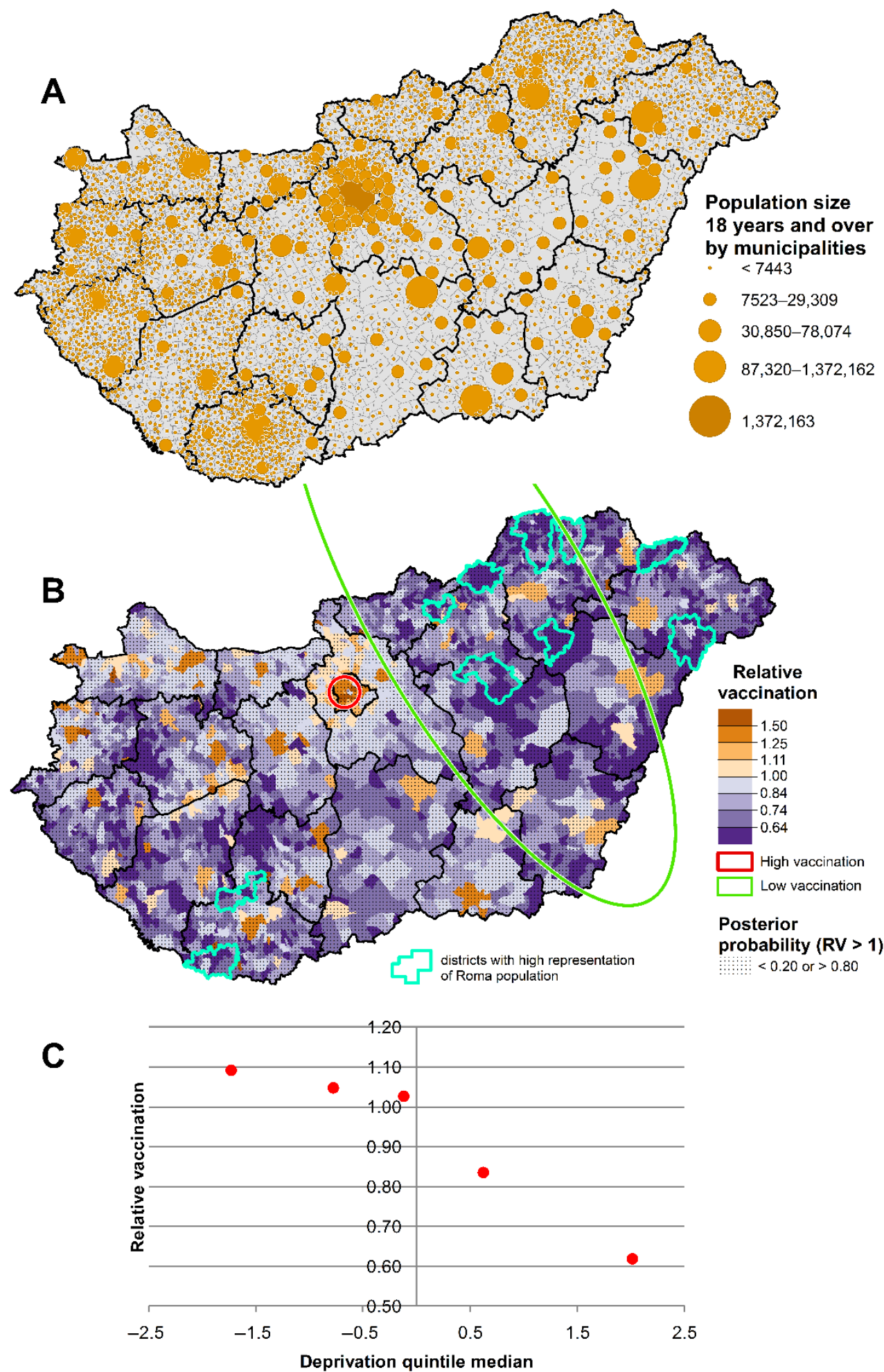

Figure 4. The spatial inequalities in population density (A), relative vaccination coverage against COVID-19 (B) and the relationship between the deprivation and vaccination coverage against COVID19 (C) by Deprivation Index quintiles during the third pandemic wave in Hungary. 
The risk analysis showed a significant inverse relationship between vaccination coverage and deprivation $\left(\chi^{2}\right.$ Homogeneity $=95,849.26, p=0 ; \chi^{2}$ Linearity $=81,178.63$, $p=0$ ) (Figure 5C). Areas of highest deprivation (V. quintile) experienced the lowest relative vaccination ratio (Relative Vaccination Ratio both sexes, V·quint.: 0.618 (CI: 0.616-0.620)) (Figure 4B,C, Table 1). In contrast, in the least deprived (I. quintile) vaccination coverage was 9\% higher (Table 1). In the municipalities in the 10 districts with the highest proportion of Roma population, the vaccination rate was only $55 \%$ of the national rate (Relative

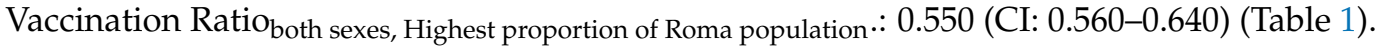

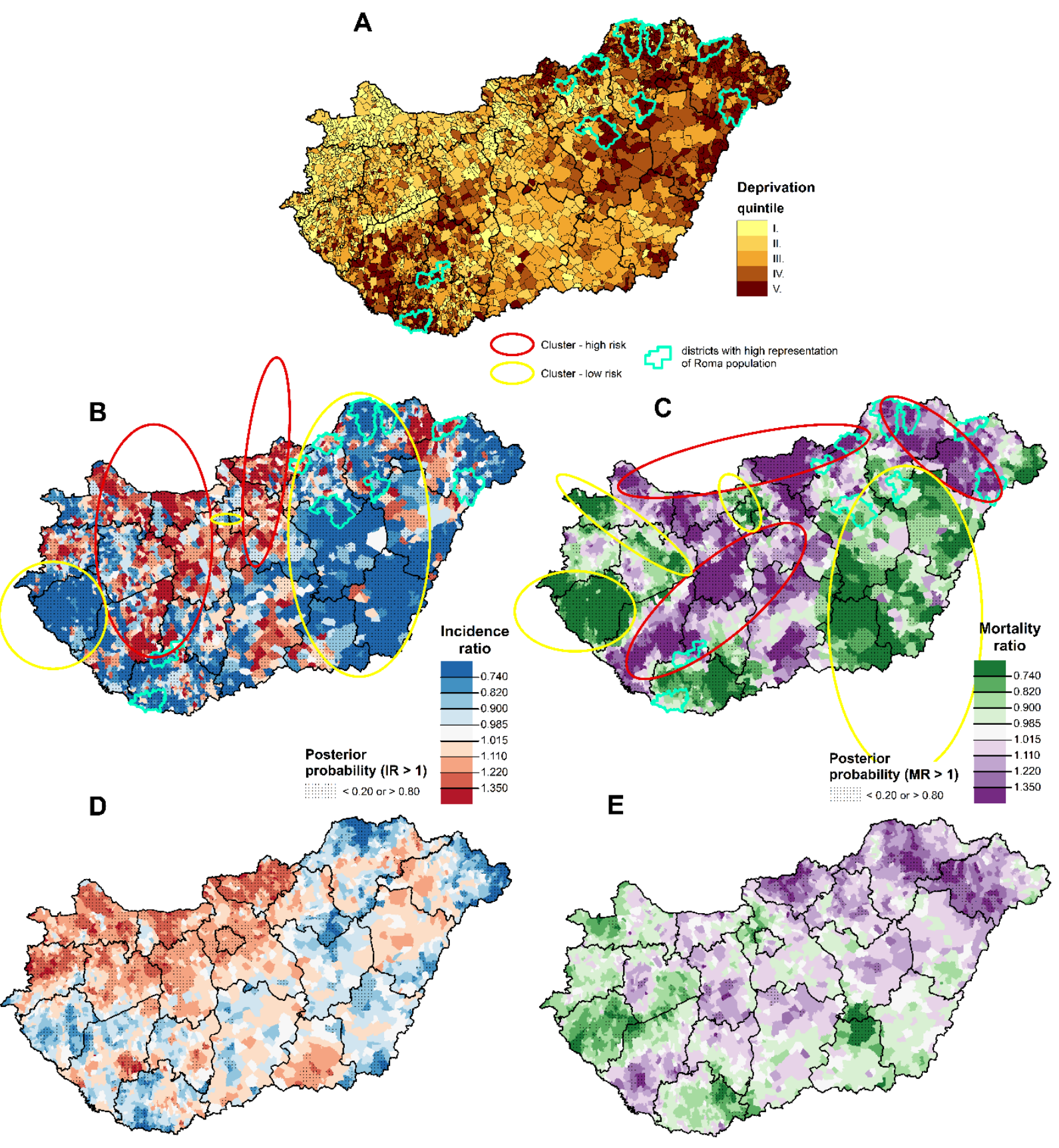

Figure 5. The spatial distribution of deprivation (A), incidence ratio of confirmed cases (B), relative mortality (C) due to COVID-19, during the third pandemic wave and the shared component of the second and third pandemic wave incidence (D) and mortality (E) in Hungary. 
Table 1. Relative vaccination coverage ratio against COVID-19 by Deprivation Index quintiles and in the districts with the highest representation of Roma during the third pandemic wave in Hungary.

\begin{tabular}{cc}
\hline DI Quintiles & Relative Vaccination Coverage Ratio \\
\hline I. (least deprived) & $1.092[1.090-1.095]$ \\
II. & $1.047[1.045-1.049]$ \\
III. & $1.026[1.024-1.028]$ \\
IV. & $0.834[0.833-0.836]$ \\
V. (most deprived) & $0.618[0.616-0.620]$ \\
Districts with highest representation of & $0.550[0.560-0.640]$ \\
Roma population & \\
\hline
\end{tabular}

Spatial inequalities of deprivation, incidence ratio, and relative mortality due to COVID-19 at the municipality level are illustrated in Figure 5.

The most deprived areas, measured by DI, are in the north-eastern and south-western parts of Hungary, and the least-deprived municipalities are in the north-western parts, as well as Budapest and neighboring areas (Figure 5A). The third wave of the COVID-19 pandemic did not affect these different areas in the same way.

A high incidence ratio of registered cases was recorded in the north-western and mid-west parts, as well as in some parts in the northeast and south (Figure 5B). High mortality ratios were recorded on the north-eastern border and in the central and mid-west parts (Figure 5C). Areas of lower incidence and lower mortality risk were concentrated in the middle of the eastern part and southwestern border (Figure 5B,C).

The shared component analysis revealed similarities in spatial patterns of incidence and mortality of the second and third pandemic wave (Figure 5D,E). Significantly higher incidence ratios were observed in both waves in the north-western quarter of Hungary, including the capital city (Figure 5D), while higher mortality risks were seen in the northeastern, north-central, central, and south-western parts of the country (Figure 5E).

The risk analysis found a significant inverse relationship between relative incidence of confirmed cases (Males: $\chi^{2}$ Homogeneity $=1575.8, p=0 ; \chi^{2}$ Linearity $=1119.51, p=0$; Females: $\chi^{2}$ Homogeneity $=603.72, p=0 ; \chi^{2}$ Linearity $=341.26, p=0$ ), and strong positive association between the relative mortality and deprivation (Males: $\chi^{2}$ Homogeneity $=82.15, p=0$; $\chi^{2}$ Linearity $=72.48, p=0$; Females: $\chi^{2}$ Homogeneity $=115.36, p=0 ; \chi^{2}$ Linearity $=111.95$, $p=0$ ) (Figure 6).
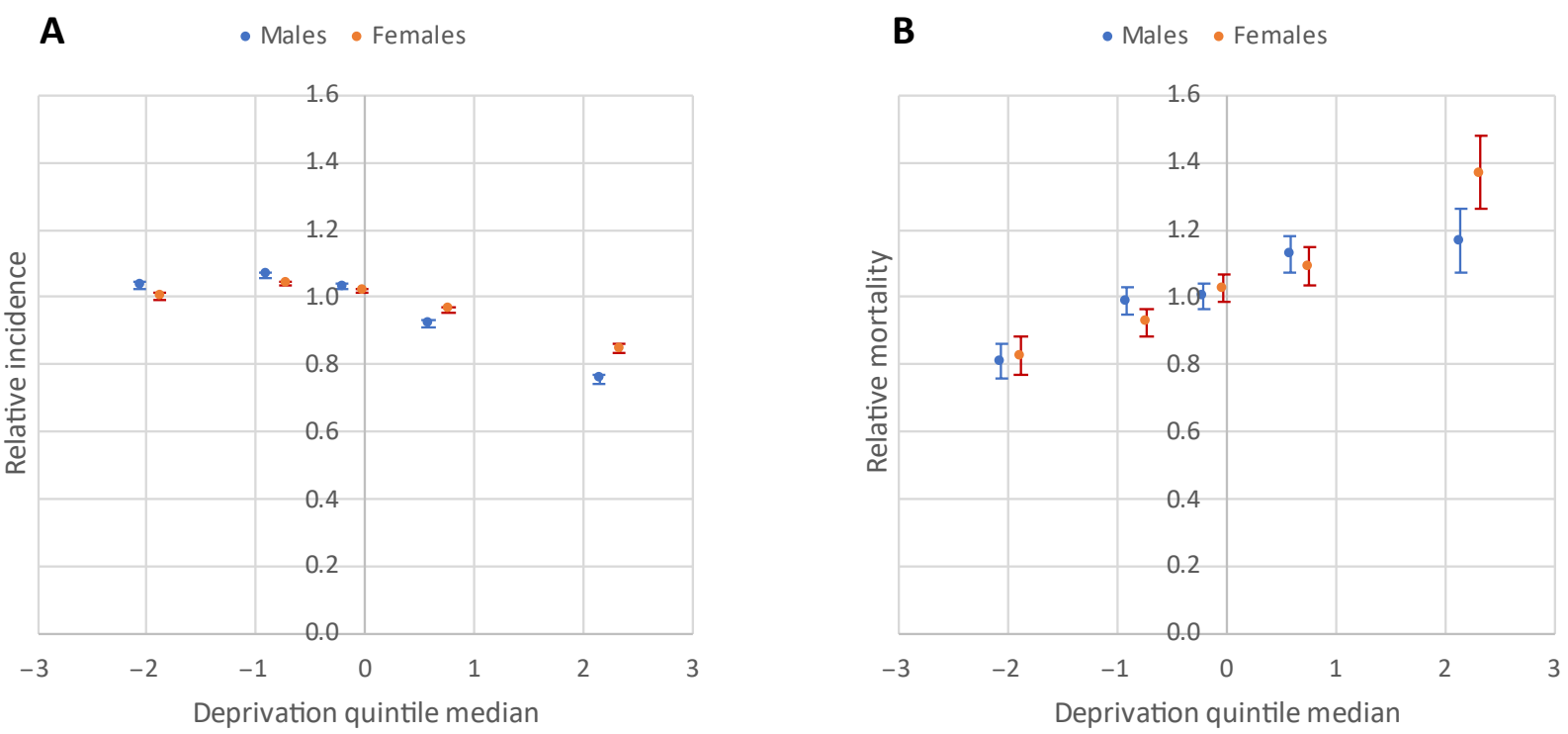

Figure 6. Relationship between the deprivation and relative incidence of confirmed cases (A), relative mortality (B) due to COVID-19 by DI quintile and sex during the third pandemic wave in Hungary. 
Areas with highest deprivation (V. quintile) had significantly lower relative incidence ratios of confirmed cases (relative incidence ratio males, V. quint.: 0.76 (CI: 0.74-0.77); relative incidence ratio females, V. quint.: 0.85 (CI: 0.83-0.86)) for both sexes (Figure 5A, Table 2). However, those people experienced the highest mortality (Relative Mortality males, $\mathrm{V} \cdot$ quint.$:$ 1.17 (CI: 1.07-1.26); Relative Mortality females, V. quint.: 1.37 (CI: 1.26-1.48)) (Figure 5B, Table 2). Overall, in quintile V (most deprived), the relative incidence was 15-24\% lower, while the relative mortality was $17-37 \%$ higher than the national average (Table 2 ).

Table 2. Relative risk of morbidity and mortality due to COVID-19 by DI quintiles during the third pandemic wave in Hungary.

\begin{tabular}{|c|c|c|c|c|}
\hline DI Quintiles & Confirmed Cases & Relative Incidence Ratio & Death Cases & Relative Mortality Ratio \\
\hline \multicolumn{5}{|l|}{ Males } \\
\hline I. (least deprived) & 31,150 & $1.03[1.02-1.05]$ & 1003 & $0.81[0.76-0.86]$ \\
\hline II. & 63,455 & $1.07[1.06-1.08]$ & 2365 & $0.99[0.95-1.03]$ \\
\hline III. & 68,449 & $1.03[1.02-1.04]$ & 2581 & $1.00[0.96-1.04]$ \\
\hline IV. & 35,831 & $0.92[0.91-0.93]$ & 1618 & $1.13[1.07-1.18]$ \\
\hline V. (most deprived) & 12,284 & $0.76[0.74-0.77]$ & 587 & $1.17[1.07-1.26]$ \\
\hline $\begin{array}{l}\text { Districts with highest } \\
\text { representation of } \\
\text { Roma population }\end{array}$ & 4526 & $0.81[0.79-0.83]$ & 230 & $1.27[1.12-1.45]$ \\
\hline \multicolumn{5}{|l|}{ Females } \\
\hline I. (least deprived) & 33,719 & $1.00[0.99-1.01]$ & 852 & $0.82[0.77-0.88]$ \\
\hline II. & 70,667 & 1.04 [1.03-1.05] & 2013 & $0.93[0.89-0.97]$ \\
\hline III. & 76,691 & $1.02[1.01-1.03]$ & 2422 & $1.03[0.99-1.07]$ \\
\hline IV. & 41,243 & $0.96[0.95-0.97]$ & 1440 & 1.09 [1.04-1.15] \\
\hline V. (most deprived) & 14,477 & $0.85[0.83-0.86]$ & 613 & $1.37[1.26-1.48]$ \\
\hline $\begin{array}{l}\text { Districts with highest } \\
\text { representation of } \\
\text { Roma population }\end{array}$ & 5550 & $0.92[0.90-0.95]$ & 255 & $1.53[1.35-1.73]$ \\
\hline
\end{tabular}

In municipalities of the 10 districts with the highest representation of Roma population, the mortality rate was $27 \%$ higher for men and $53 \%$ higher for women than the national rate (Relative Mortality males, Highest representation of Roma pop.: 1.27 (CI: 1.12-1.45); Relative Mortality females, Highest representation of Roma pop.: 1.53 (CI: 1.35-1.73)) (Figure 5C, Table 2).

In contrast, in the least deprived quintile, the incidence was only $3 \%$ higher than the national average for males, and it was similar to the national average for women, but mortality was almost $20 \%$ lower than the national average in both sexes.

Relative risk of morbidity and mortality due to COVID-19 by DI quintiles during the third pandemic wave in Hungary. The ecological regression showed an inverse association between vaccination and mortality $\left(R_{\text {Vaccination }}=0.86(0.75-0.98)\right)$, while deprivation had a strong positive association with mortality. However, the former became non-significant after adjusting for deprivation $\left(R R_{\text {Vaccination }}=0.95(0.84-1.09), R_{\text {Deprivation }}=1.10(1.07-1.14)\right.$ ).

\section{Discussion}

Despite having a complex package of nonpharmacological measures in place and a rapidly expanding vaccination campaign, one of the fastest in the EU with $24.4 \%$ of the population receiving at least one dose of COVID-19 vaccine by the time the third pandemic wave peaked and $52.5 \%$ fully vaccinated when it ended, Hungary was unable to contain the third pandemic wave, caused by the more virulent and faster spreading SARS-CoV-2 Alpha variant. Thus, the third wave exceeded the size of the second wave in terms of both the number of cases registered and the number of COVID-19-related deaths [42,43]. In addition, the premature excess mortality for the 50-64-year-old population was markedly higher for the third wave than in the second wave [18]. 
Hungary's experience is a reminder of the need to look beyond the aggregate data. Although the vaccination campaign started before the third wave, differences in vaccine acceptance soon emerged, initially unnoticed. Less deprived, mainly urban areas achieved higher coverage so, by the end of the third wave, protection was strongly socially patterned. This is consistent with experience elsewhere, in the UK and the United States $[12,13,44,45]$. As expected, coverage was especially low in areas with the highest proportion of Roma population, even more so than in the other most deprived areas.

To our knowledge, our study is one of the first to investigate the health impact of a COVID-19 vaccination program within a country during a pandemic wave, incorporating data on both socioeconomic inequalities and vaccination. As expected, we found an inverse association between vaccination and mortality, with the latter, again, as expected, being strongly associated with deprivation. After adjusting for deprivation as a confounder, there was no evidence of an association between municipal level vaccination coverage and the spatial pattern of mortality. This seems counterintuitive, given the clear evidence of the effectiveness of the vaccines.

Concerning the fact that $38.7 \%$ of the population fully vaccinated had received a vaccine with no European Medicines Agency authorization but emergency approval in Hungary (Sputnik V or Sinopharm), it is reasonable to suppose that the type of vaccine administered might influence our results. In the present study, this question was not investigated, but it seems very likely that the distribution of different vaccines administered did not influence our results significantly, which is supported by the results of a nationwide, retrospective observational study (conducted between 22 January and 10 June 2021 in Hungary), which showed similarly high effectiveness of the different two-dose vaccines used in Hungary. Regarding morbidity among fully vaccinated people, incidence rates varied in a relatively narrow range-between 0.04 (Sputnik V) and 0.6 (Sinopharm) per 100,000 person days - in the relevant fully vaccinated populations [46]. In line with the Hungarian data in a community-based, retrospective, observational study carried out in the United Arab Emirates, it was shown that Sinopharm vaccine effectiveness in fully vaccinated individuals was $80 \%, 92 \%$, and $97 \%$ in preventing COVID-19-related hospital admissions, critical care admissions, and death, respectively [47]. A recent narrative review based on the comparative analysis of the characteristics, adverse events, efficacy, effectiveness, and impact of 19 variants (including Sputnik V and Sinopharm) of COVID-19 vaccines also concludes that "all vaccines appear to be safe and effective tools to prevent severe COVID-19, hospitalization, and death", although BNT162b2, mRNA-1273 and Sputnik V after two doses had the highest efficacy ( $>90 \%)$. As a limitation of this narrative review, it was mentioned that large observational studies were lacking for several authorized vaccines and, even if they were carried out, are subject to bias when assessing effectiveness, such as misclassification from diagnostic errors, imbalances in socioeconomic status, exposure risk, healthcare-seeking behaviors, or immunity status between vaccinated and unvaccinated groups [48].

However, there are several plausible explanations. First, although the Hungarian vaccine roll out was fast, the spread of the pandemic was even faster, and it took time to reach high numbers of people who had two doses. Secondly, and assuming patterns observed elsewhere apply equally to Hungary, those individuals and households that are most disadvantaged within a given area are most likely to become infected and severely ill and be unvaccinated.

The deprivation index is a multidimensional index that considers and combines several socioeconomic factors, including educational level. Several studies have confirmed that education significantly influences the willingness to accept vaccination, as those with lower education are more likely to refuse COVID-19 vaccination [49-53]. Since education is one of the most important (if not the most important) determinants of the deprivation index [20], it is assumed that higher education and the related higher willingness to accept vaccination may be a decisive factor for the higher vaccination coverage identified in more advantaged socioeconomic areas. In addition, in a recent study covering 41 countries, it 
was clearly shown that low levels of educational attainment (in $66 \%$ of countries) and low income $(80 \%)$ were also positively correlated with non-compliance with the recommendations/restrictions [54].

This study adds to the literature by describing the epidemiology of COVID-19 incidence at a granular level within a country where it can be related to the socioeconomic situation. It is striking that both waves significantly affected the north-western quarter of the country and the capital, as measured by recorded COVID-19 cases, but these areas had a relative mortality lower than the national average. In contrast, the north-eastern parts of the country were hit hard in terms of mortality in both waves, but this would not have been expected on the basis of recorded case numbers. Thus, the highest morbidity was in the least deprived areas, but the highest mortality was in the most deprived areas. This was also found in the second wave [18]. Additionally, as seen in the second wave, areas with the highest proportion of Roma population had mortality rates considerably higher than the national rate, more than could be explained by the aggregate measure of deprivation.

The persistence of these patterns points to the role of unmeasured factors, likely related to inequalities in testing, contact tracing, access to health care, and in risk-avoiding behaviors.

Concerning the districts with the highest representation of Roma population, a higher relative mortality ratio was identified for Roma females than for Roma males, but because of the different sex-specific reference rates, the relative mortality ratio for females is not directly comparable for males. However, considering 95\% confidence intervals around the point estimates, the magnitude of the deviation from the sex-specific references, which indicates the degree of inequality, does not differ significantly between Roma men and women.

This study is subject to several limitations. No spatial analysis can capture all relevant individual risk factors, such as underlying health conditions. Ascertainment of cases is influenced by access to testing, willingness to test and to be tested, and possibly enhanced surveillance of certain groups, such as those with chronic illness or a higher threshold for testing in those presumed to be at less risk having been vaccinated. Our findings point to a persistence of undertesting among those living in the most disadvantaged areas in both pandemic waves [18]. The lower incidence rates in the more deprived areas do not necessarily mean that there are fewer COVID-19 infections, but they may indicate underdiagnosis due to less testing. This hypothesis cannot be verified, because data about the number of COVID-19 tests performed at the municipality level are not available in Hungary. COVID-19 testing is available both free of charge and for a fee in Hungary. However, access and availability to free testing and, in particular, fee-based testing may have been influenced and restricted by socioeconomic status. Ascertainment of COVIDrelated death can be also biased due to geographical differences in the determination of causes of death but, given the duration of the pandemic, the importance of these problems may have diminished.

Assuming that biases and uncontrolled confounding have remained constant, our results concerning COVID-19 morbidity and mortality can be compared over time. Vaccination data may be incomplete, which might cause some underestimation of the coverage data over time.

Another limitation of the study is the limited timeliness of the deprivation index. Most of the indicators used for the index are only available from the 2011 census data and, therefore, as for all such area-based socioeconomic indicators, the accuracy of the index diminishes over time. It should be noted, however, that the spatial distribution of the indices based on the 2001 and 2011 census data, compiled with the same methodology (although not directly comparable in terms of values), did not show any significant, marked change and the deprived areas showed a nearly identical spatial distribution within Hungary, which suggests that the socioeconomic characteristic at the territorial level is not changing, or if so, only very slowly $[18,20]$.

In conclusion, despite the availability of several effective vaccines, the Hungarian vaccination program faced difficulties in reaching those in the most disadvantaged areas. The ecological regression showed that the uneven coverage by the vaccination program 
was inadequate to overcome this disadvantage and more effort was needed to reach certain groups, who were likely at greatest risk of COVID-19. We have safe and effective vaccines, but they can only work if they reach those at greatest need.

Author Contributions: Conceptualization, B.O., A.J., C.N. and R.Á., M.M.; Methodology, B.O., A.J., C.N., J.K.H. and R.Á.; Validation, J.K.H., B.O.; Formal Analysis, A.J., C.N., K.E.K., G.T.; Investigation, A.J., C.N., J.K.H., K.E.K., G.T.; Data Curation, J.K.H., K.E.K.; Writing-Original Draft Preparation, B.O., A.J., C.N., J.K.H.; Writing—Review and Editing, R.Á., M.M.; Visualization, A.J., K.E.K.; Supervision, R.Á., M.M. All authors have read and agreed to the published version of the manuscript.

Funding: This study was funded by the Hungarian Academy of Sciences (TK2016-78) and project No. 135784 has also been implemented with the support provided from the National Research, Development and Innovation Fund of Hungary, financed under the K_20 funding scheme.

Institutional Review Board Statement: Not applicable.

Informed Consent Statement: Not applicable.

Data Availability Statement: All relevant data may be obtained from the corresponding author upon a reasonable request and prior permission of the study funder.

Acknowledgments: The authors thank János Pénzes (Department of Social Geography and Regional Development Planning, Institute of Earth Sciences, Faculty of Science and Technology, University of Debrecen) for providing data on the Roma population.

Conflicts of Interest: The authors declare no conflict of interest.

\section{References}

1. Davies, N.G.; Kucharski, A.J.; Eggo, R.M.; Gimma, A.; Edmunds, W.J. Centre for the Mathematical Modelling of Infectious Diseases COVID-19 working group Effects of Non-Pharmaceutical Interventions on COVID-19 Cases, Deaths, and Demand for Hospital Services in the UK: A Modelling Study. Lancet Public Health 2020, 5, e375-e385. [CrossRef]

2. ECDC. Guidelines for the Implementation of Non-Pharmaceutical Interventions against COVID-19. Available online: https: / / www.ecdc.europa.eu/en/publications-data/covid-19-guidelines-non-pharmaceutical-interventions (accessed on 19 January 2022).

3. Hall, V.J.; Foulkes, S.; Saei, A.; Andrews, N.; Oguti, B.; Charlett, A.; Wellington, E.; Stowe, J.; Gillson, N.; Atti, A.; et al. COVID-19 Vaccine Coverage in Health-Care Workers in England and Effectiveness of BNT162b2 MRNA Vaccine against Infection (SIREN): A Prospective, Multicentre, Cohort Study. Lancet 2021, 397, 1725-1735. [CrossRef]

4. Lopez Bernal, J.; Andrews, N.; Gower, C.; Robertson, C.; Stowe, J.; Tessier, E.; Simmons, R.; Cottrell, S.; Roberts, R.; O'Doherty, M.; et al. Effectiveness of the Pfizer-BioNTech and Oxford-AstraZeneca Vaccines on COVID-19 Related Symptoms, Hospital Admissions, and Mortality in Older Adults in England: Test Negative Case-Control Study. BMJ 2021, 373 , n1088. [CrossRef]

5. Haas, E.J.; Angulo, F.J.; McLaughlin, J.M.; Anis, E.; Singer, S.R.; Khan, F.; Brooks, N.; Smaja, M.; Mircus, G.; Pan, K.; et al. Impact and Effectiveness of MRNA BNT162b2 Vaccine against SARS-CoV-2 Infections and COVID-19 Cases, Hospitalisations, and Deaths Following a Nationwide Vaccination Campaign in Israel: An Observational Study Using National Surveillance Data. Lancet 2021, 397, 1819-1829. [CrossRef]

6. Tartof, S.Y.; Slezak, J.M.; Fischer, H.; Hong, V.; Ackerson, B.K.; Ranasinghe, O.N.; Frankland, T.B.; Ogun, O.A.; Zamparo, J.M.; Gray, S.; et al. Effectiveness of MRNA BNT162b2 COVID-19 Vaccine up to 6 Months in a Large Integrated Health System in the USA: A Retrospective Cohort Study. Lancet 2021, 398, 1407-1416. [CrossRef]

7. Moore, S.; Hill, E.M.; Tildesley, M.J.; Dyson, L.; Keeling, M.J. Vaccination and Non-Pharmaceutical Interventions for COVID-19: A Mathematical Modelling Study. Lancet Infect. Dis. 2021, 21, 793-802. [CrossRef]

8. Pung, R.; Firth, J.A.; Spurgin, L.G. Singapore CruiseSafe working group; CMMID COVID-19 working group; Vernon J Lee; Adam J Kucharski Using High-Resolution Contact Networks to Evaluate SARS-CoV-2 Transmission and Control in Large-Scale Multi-Day Events. medRxiv 2021. [CrossRef]

9. Sonabend, R.; Whittles, L.K.; Imai, N.; Perez-Guzman, P.N.; Knock, E.S.; Rawson, T.; Gaythorpe, K.A.M.; Djaafara, B.A.; Hinsley, W.; FitzJohn, R.G.; et al. Non-Pharmaceutical Interventions, Vaccination, and the SARS-CoV-2 Delta Variant in England: A Mathematical Modelling Study. Lancet 2021, 398, 1825-1835. [CrossRef]

10. Betti, M.; Bragazzi, N.L.; Heffernan, J.M.; Kong, J.; Raad, A. Integrated Vaccination and Non-Pharmaceutical Interventions Based Strategies in Ontario, Canada, as a Case Study: A Mathematical Modelling Study. J. R. Soc. Interface 2021, 18, 20210009. [CrossRef]

11. Douglas, M.; Katikireddi, S.V.; Taulbut, M.; McKee, M.; McCartney, G. Mitigating the Wider Health Effects of COVID-19 Pandemic Response. BMJ 2020, 369, m1557. [CrossRef] 
12. Perry, M.; Akbari, A.; Cottrell, S.; Gravenor, M.B.; Roberts, R.; Lyons, R.A.; Bedston, S.; Torabi, F.; Griffiths, L. Inequalities in Coverage of COVID-19 Vaccination: A Population Register Based Cross-Sectional Study in Wales, UK. Vaccine 2021, 39, 6256-6261. [CrossRef] [PubMed]

13. Gaddis, S.M.; Carey, C.M.; DiRago, N.V. Changes Over Time in COVID-19 Vaccination Inequalities in Eight Large U.S. Cities. medRxiv 2021. [CrossRef]

14. Boehm, E.; Kronig, I.; Neher, R.A.; Eckerle, I.; Vetter, P.; Kaiser, L.; Geneva Centre for Emerging Viral Diseases. Novel SARS-CoV-2 Variants: The Pandemics within the Pandemic. Clin. Microbiol Infect. 2021, 27, 1109-1117. [CrossRef] [PubMed]

15. Tallon, J.M.; Gomes, P.; Bacelar-Nicolau, L.; Bacelar, S. A Three-Way Multivariate Data Analysis: Comparison of EU Countries' COVID-19 Incidence Trajectories from May 2020 to February 2021. Biom. Biostat. Int. J. 2021, 10, 98-114. [CrossRef]

16. ECDC. COVID-19 Data Explorer. Available online: https://www.ecdc.europa.eu/en/publications-data/data-covid-19 -vaccination-eu-eea (accessed on 31 January 2022).

17. COVID-19 Data Explorer. Available online: https://ourworldindata.org/coronavirus-data-explorer (accessed on 3 February 2022).

18. Oroszi, B.; Juhász, A.; Nagy, C.; Horváth, J.K.; McKee, M.; Ádány, R. Unequal Burden of COVID-19 in Hungary: A Geographical and Socioeconomic Analysis of the Second Wave of the Pandemic. BMJ Glob. Health 2021, 6, e006427. [CrossRef] [PubMed]

19. National Public Health Center Procedures for the New Coronavirus Disease Identified in 2020. 2021. Available online: https://koronavirus.gov.hu/sites/default/files/sites/default/files/imce/nnk_eljarasrend_2021_oktober.pdf (accessed on 21 November 2021).

20. Juhász, A.; Nagy, C.; Páldy, A.; Beale, L. Development of a Deprivation Index and Its Relation to Premature Mortality Due to Diseases of the Circulatory System in Hungary, 1998-2004. Soc. Sci. Med. 2010, 70, 1342-1349. [CrossRef] [PubMed]

21. Juhász, A.; Nagy, C.; Varga, O.; Boruzs, K.; Csernoch, M.; Szabó, Z.; Ádány, R. Antithrombotic Preventive Medication Prescription Redemption and Socioeconomic Status in Hungary in 2016: A Cross-Sectional Study. Int. J. Environ. Res. Public Health 2020, 17, 6855. [CrossRef]

22. Nagy, C.; Juhász, A.; Beale, L.; Páldy, A. Mortality Amenable to Health Care and Its Relation to Socio-Economic Status in Hungary, 2004-2008. Eur. J. Public Health 2012, 22, 620-624. [CrossRef]

23. Nagy, C.; Juhász, A.; Pikó, P.; Diószegi, J.; Paragh, G.; Szabó, Z.; Varga, O.; Ádány, R. Preventive Metformin Monotherapy Medication Prescription, Redemption and Socioeconomic Status in Hungary in 2018-2019: A Cross-Sectional Study. Int. J. Environ. Res. Public Health 2021, 18, 2206. [CrossRef]

24. Boruzs, K.; Juhász, A.; Nagy, C.; Ádány, R.; Bíró, K. Relationship between Statin Utilization and Socioeconomic Deprivation in Hungary. Front. Pharmacol. 2016, 7, 66. [CrossRef]

25. Jakab, Z.; Juhasz, A.; Nagy, C.; Schuler, D.; Garami, M.; Hungarian Paediatric Haemato-Oncology Network. Trends and Territorial Inequalities of Incidence and Survival of Childhood Leukaemia and Their Relations to Socioeconomic Status in Hungary, 1971-2015. Eur. J. Cancer Prev. 2017, 26, S183-S190. [CrossRef] [PubMed]

26. Papp, M.; Kőrösi, L.; Sándor, J.; Nagy, C.; Juhász, A.; Ádány, R. Workforce Crisis in Primary Healthcare Worldwide: Hungarian Example in a Longitudinal Follow-up Study. BMJ Open 2019, 9, e024957. [CrossRef] [PubMed]

27. Beale, L.; Hodgson, S.; Abellan, J.J.; LeFevre, S.; Jarup, L. Evaluation of Spatial Relationships between Health and the Environment: The Rapid Inquiry Facility. Environ. Health Perspect. 2010, 118, 1306-1312. [CrossRef]

28. Besag, J.; York, J.; Mollié, A. Bayesian Image Restoration, with Two Applications in Spatial Statistics. Ann. Inst. Stat. Math. 1991, 43, 1-20. [CrossRef]

29. Rue, H.; Martino, S.; Chopin, N. Approximate Bayesian Inference for Latent Gaussian Models by Using Integrated Nested Laplace Approximations. J. R. Stat. Soc. 2009, 71, 319-392. [CrossRef]

30. Richardson, S.; Thomson, A.; Best, N.; Elliott, P. Interpreting Posterior Relative Risk Estimates in Disease-Mapping Studies. Env. Health Perspect. 2004, 112, 1016-1025. [CrossRef]

31. Kulldorff, M.; Nagarwalla, N. Spatial Disease Clusters: Detection and Inference. Stat. Med. 1995, 14, 799-810. [CrossRef]

32. Pénzes, J.; Pásztor, I.Z.; Tátrai, P.; Kóti, T. Roma Population in Hungary-Spatial Distribution and Its Temporal Changes. Deturope Cent. Eur. J. Tour. Reg. Dev. 2019, 11, 138-159. [CrossRef]

33. Pénzes, J.; Tátrai, P.; Pásztor, I.Z. Changes in the Spatial Distribution of the Roma Population in Hungary during the Last Decades. Területi. Stat. 2018, 58, 3-26.

34. Knorr-Held, L.; Best, N.G. A Shared Component Model for Detecting Joint and Selective Clustering of Two Diseases. J. R. Stat. Society. Ser. A 2001, 164, 73-85. [CrossRef]

35. Best, N.; Hansell, A.L. Geographic Variations in Risk: Adjusting for Unmeasured Confounders through Joint Modeling of Multiple Diseases. Epidemiology 2009, 20, 400-410. [CrossRef] [PubMed]

36. Lunn, D.J.; Thomas, A.; Best, N.; Spiegelhalter, D. WinBUGS-A Bayesian Modelling Framework: Concepts, Structure, and Extensibility. Stat. Comput. 2000, 10, 325-337. [CrossRef]

37. Brooks, S.P.; Gelman, A. General Methods for Monitoring Convergence of Iterative Simulations. J. Comput. Graph. Stat. 1998, 7, 434-455.

38. Schabenberger, O.; Gotway, C.A. Statistical Methods for Spatial Data Analysis; CRC Press: Boca Raton, FL, USA, 2004; ISBN 978-0-203-49198-0. 
39. Belsley, D.A.; Kuh, E.; Welsch, R.E. Regression Diagnostics: Identifying Influential Data and Sources of Collinearity; John Wiley \& Sons: New York, NY, USA, 2005.

40. Spiegelhalter, D.J.; Best, N.G.; Carlin, B.P. Bayesian Measures of Model Complexity and Fit. J. R. Stat. Soc. Ser. B 2002, 64, 583-639. [CrossRef]

41. Gelman, A.; Hwang, J.; Vehtari, A. Understanding Predictive Information Criteria for Bayesian Models. Stat. Comput. 2014, 24, 997-1016. [CrossRef]

42. ECDC. Risk of Spread of New SARS-CoV-2 Variants of Concern in the EU/EEA-First Update 2021. Available online: https:/ / www.ecdc.europa.eu/sites / default/ files/documents/COVID-19-risk-related-to-spread-of-new-SARS-CoV-2variants-EU-EEA-first-update.pdf (accessed on 21 November 2021).

43. Grint, D.J.; Wing, K.; Houlihan, C.; Gibbs, H.P.; Evans, S.J.W.; Williamson, E.; McDonald, H.I.; Bhaskaran, K.; Evans, D.; Walker, A.J.; et al. Severity of SARS-CoV-2 Alpha Variant (B.1.1.7) in England. Clin. Infect. Dis. 2021. [CrossRef]

44. Barry, V.; Dasgupta, S.; Weller, D.L.; Kriss, J.L.; Cadwell, B.L.; Rose, C.; Pingali, C.; Musial, T.; Sharpe, J.D.; Flores, S.A.; et al. Patterns in COVID-19 Vaccination Coverage, by Social Vulnerability and Urbanicity-United States, December 14, 2020-May 1, 2021. MMWR Morb. Mortal. Wkly. Rep. 2021, 70, 818-824. [CrossRef]

45. Hughes, M.M.; Wang, A.; Grossman, M.K. County-Level COVID-19 Vaccination Coverage and Social Vulnerability-United States, December 14, 2020-March 1, 2021. MMWR Morb. Mortal. Wkly. Rep. 2021, 70, 818-824. [CrossRef]

46. Vokó, Z.; Kiss, Z.; Surján, G.; Surján, O.; Barcza, Z.; Pályi, B.; Formanek-Balku, E.; Molnár, G.A.; Herczeg, R.; Gyenesei, A.; et al. Nationwide Effectiveness of Five SARS-CoV-2 Vaccines in Hungary-the HUN-VE Study. Clin. Microbiol. Infect. 2021. [CrossRef]

47. Ismail AlHosani, F.; Eduardo Stanciole, A.; Aden, B.; Timoshkin, A.; Najim, O.; Abbas Zaher, W.; AlSayedsaleh AlDhaheri, F.; Al Mazrouie, S.; Rizvi, T.A.; Mustafa, F. Impact of the Sinopharm's BBIBP-CorV Vaccine in Preventing Hospital Admissions and Death in Infected Vaccinees: Results from a Retrospective Study in the Emirate of Abu Dhabi, United Arab Emirates (UAE). Vaccine 2022. [CrossRef]

48. Fiolet, T.; Kherabi, Y.; MacDonald, C.-J.; Ghosn, J.; Peiffer-Smadja, N. Comparing COVID-19 Vaccines for Their Characteristics, Efficacy and Effectiveness against SARS-CoV-2 and Variants of Concern: A Narrative Review. Clin. Microbiol. Infect. 2022, 28, 202-221. [CrossRef] [PubMed]

49. Kessels, R.; Luyten, J.; Tubeuf, S. Willingness to Get Vaccinated against COVID-19 and Attitudes toward Vaccination in General. Vaccine 2021, 39, 4716-4722. [CrossRef] [PubMed]

50. Nehal, K.R.; Steendam, L.M.; Campos Ponce, M.; van der Hoeven, M.; Smit, G.S.A. Worldwide Vaccination Willingness for COVID-19: A Systematic Review and Meta-Analysis. Vaccines 2021, 9, 1071. [CrossRef] [PubMed]

51. Rodríguez-Blanco, N.; Montero-Navarro, S.; Botella-Rico, J.M.; Felipe-Gómez, A.J.; Sánchez-Más, J.; Tuells, J. Willingness to Be Vaccinated against COVID-19 in Spain before the Start of Vaccination: A Cross-Sectional Study. Int. J. Environ. Res. Public Health 2021, 18, 5272. [CrossRef]

52. Syan, S.K.; Gohari, M.R.; Levitt, E.E.; Belisario, K.; Gillard, J.; DeJesus, J.; MacKillop, J. COVID-19 Vaccine Perceptions and Differences by Sex, Age, and Education in 1367 Community Adults in Ontario. Front. Public Health 2021. [CrossRef]

53. Wang, B.; Nolan, R.; Marshall, H. COVID-19 Immunisation, Willingness to Be Vaccinated and Vaccination Strategies to Improve Vaccine Uptake in Australia. Vaccines 2021, 9, 1467. [CrossRef]

54. Szaszi, B.; Hajdu, N.; Szecsi, P.; Tipton, E.; Aczel, B. A Machine Learning Analysis of the Relationship of Demographics and Social Gathering Attendance from 41 Countries during Pandemic. Sci. Rep. 2022, 12, 724. [CrossRef] 